Reviews

\title{
Biological and Medical Applications of Multivariate Curve Resolution Assisted Raman Spectroscopy
}

\author{
Hemanth NoothalaPatI, ${ }^{* \dagger}$ Keita IwaSAKI, $* *$ and Tatsuyuki Yamamoto $* * * \dagger$ \\ *Raman Center for Medical and Biological Applications, Shimane University, Matsue 690-8504, Japan \\ **Faculty of Life and Environmental Science, Shimane University, Matsue 690-8504, Japan
}

\begin{abstract}
Biological specimens such as cells, tissues and biofluids (urine, blood) contain mixtures of many different biomolecules, all of which contribute to a Raman spectrum at any given point. The separation and identification of pure biochemical components remains one of the biggest challenges in Raman spectroscopy. Multivariate curve resolution, a matrix factorization method, is a powerful, yet flexible, method that can be used with constraints, such as non-negativity, to decompose a complex spectroscopic data matrix into a small number of physically meaningful pure spectral components along with their relative abundances. This paper reviews recent applications of multivariate curve resolution by alternating least squares analysis to Raman spectroscopic and imaging data obtained either in vivo or in vitro from biological and medical samples.
\end{abstract}

Keywords MCR, MCR-ALS, Raman spectroscopy, disease diagnosis, single cell biology, polysaccharide imaging

(Received August 25, 2016; Accepted November 15, 2016; Published January 10, 2017)

$\begin{array}{llll}1 \text { Introduction } & 15 & 3 \cdot 3 \text { Exploratory studies } & \\ 2 \text { Method } & 16 & 4 \text { Medical Applications } & 19 \\ 3 \text { Cellular Applications } & 17 & 5 \text { Conclusions and Outlooks } & 21 \\ 3 \cdot 1 \text { Cell wall polysaccharide imaging } & & 6 \text { Acknowledgements } & 21 \\ 3 \cdot 2 \text { Single cell biochemistry } & & 7 \text { References } & 21\end{array}$

\section{Introduction}

Raman spectroscopy is a powerful vibrational spectroscopic technique that can differentiate molecular structures based on the inelastic scattering of monochromatic light, such as laser light. A Raman spectrum, otherwise called a molecular fingerprint, therefore provides rich chemical information with high specificity, which can be used in both qualitative and quantitative analytical applications. It offers several advantages over other spectroscopic methods: 1) suitability for biological cells and tissues [water is a weak Raman scatterer]; 2) does not require any sample pretreatment [no need for external dye probes or genetic manipulations]; 3) a low or non-invasive method, and hence suitable for in vivo applications. Raman spectroscopy, when combined with a microscope, achieves sub- $\mu \mathrm{m}$ spatial resolution, which can provide detailed spaceresolved molecular information and visualize their intracellular distribution. Owing to its ability to detect biochemical changes at the molecular level, Raman spectroscopy has gained much

† To whom correspondence should be addressed. E-mail:nvhnag@life.shimane-u.ac.jp (H. N.); tyamamot@life. shimane-u.ac.jp (T. Y.) attention as a bio-analytical tool in recent years, and has been successfully applied in myriad different problems in biological and medical sciences, which include, but not limited to, structural identification and label-free biomolecular imaging of living cells, ${ }^{1-7}$ cellular metabolic pathways, ${ }^{8,9}$ cellular discrimination, ${ }^{10-13}$ tissue characterization, ${ }^{14,15}$ disease diagnosis, prognosis and therapy. ${ }^{16-20}$

Although Raman spectroscopy seems to be simple and straightforward, in principle, it is often difficult to interpret Raman spectra by the univariate method (analyzing one band at a time). Many times it is difficult to identify marker bands for a specific bio-molecular component. This limitation arises because the Raman spectrum from a given point is contributed by many molecules that give vibrational bands in the same region, leading to an overlapping of the spectral information. Additionally, auto-fluorescence (in cells and tissues), which is overwhelmingly intense, competes with Raman scattering, and often masks Raman signals. This further complicates both experiment and analysis. Therefore sophisticated data-analysis methods are needed to obtain detailed information. In order to extract underlying chemical information from such complex Raman spectra, a variety of chemometric methods that take the whole spectrum into account have been developed and applied. Of the many multivariate data analytical techniques, principal 


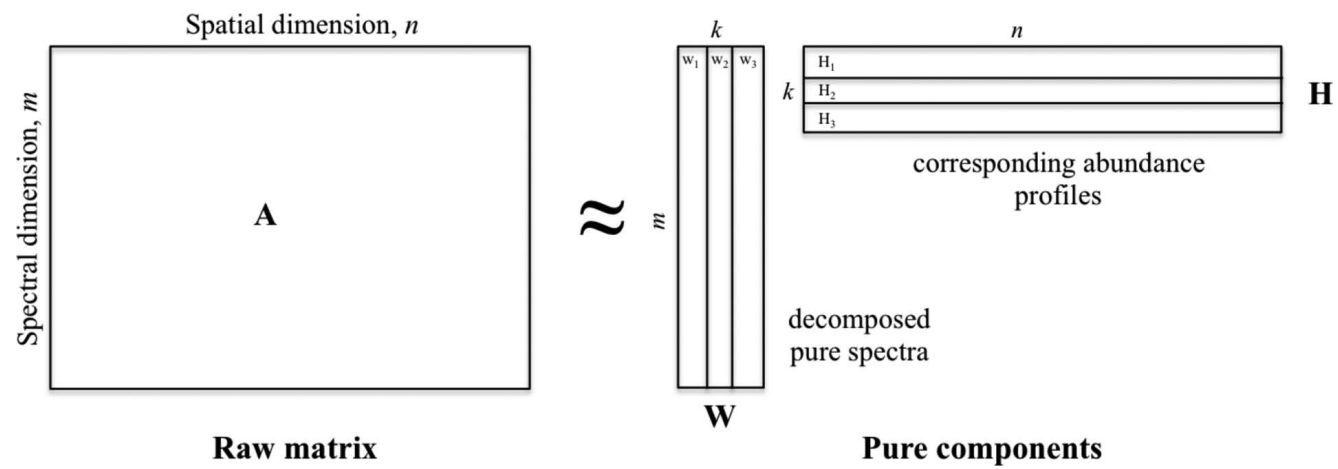

Fig. 1 Schematic representation of the principle of MCR assuming 3 components.

component analysis (PCA) and clustering analysis (CA) are two popular methods. PCA gives an orthogonal set of principal spectral components that have both positive and negative features. Though it is helpful for deducing the number of different sources of variation present in the data set and partially decomposes molecular information, the results are only abstract solutions with no physical meaning. ${ }^{21-24}$ Cluster analysis, another widely used spectral decomposition method, partitions a dataset into a pre-determined number of subgroups of similar spectra by statistically analyzing the variations. It has been successfully applied to identify intracellular structures and discriminate tissue environments. ${ }^{14,25}$ However, the identified spectral components may or may not represent pure chemical component in real terms because it is only an average of all members in the subgroup. Therefore, there is a need to develop methods that not only identify the number of components involved, but also the nature of components i.e., extract pure chemical information from raw spectrum obtained from mixtures.

Multivariate curve resolution-alternating least-squares (MCRALS) method, also known as non-negative matrix factorization or self-modeling curve resolution, is useful in this regard. MCR, a matrix factorization technique, involves linear combination of a prescribed number of spectral components under constraints, such as non-negativity to both spectral and concentration profiles. This leads to the extraction of physically meaningful spectra that represent pure chemical components without any prior information concerning the nature of the sample. ${ }^{26}$ In fact, it has already been made use of in many spectroscopic techniques, which include LC/MS, ${ }^{27-29} \mathrm{GC} / \mathrm{MS},{ }^{30-32} \mathrm{UV}-\mathrm{Vis},{ }^{33-35}$ FT-IR, ${ }^{36,37}$ NIR $^{38}$ and fluorescence spectroscopies. ${ }^{39-41}$ It is only recently that Raman spectroscopists have become interested in employing MCR to disentangle dynamic changes of multiple bio-molecular components in complex samples, like cells and tissues. This review provides an overview of applications of MCR assisted Raman spectroscopy, and focuses on some selected papers that emphasize its recent advancements, particularly in biological and medical sciences. Section 2 describes the method used to understand how MCR-ALS works. Section 3 presents some recent examples of how MCR-ALS has helped to deepen our understanding in cell biology and cellular biochemistry at the single cell level. Section 4 discusses medical applications of MCR-ALS, while particularly focusing on disease diagnostics.

\section{Method}

MCR-ALS analysis is a matrix approximation method that iteratively finds the matrices of pure component spectra and their relative abundances (distribution images). Suppose that we have a data matrix A (a $m \times n$ non-negative data matrix) containing experimental Raman spectra whose rows $m$ represent number of pixels in each spectrum (wavenumber axis) and columns $n$ represent number of spectra acquired at different measurement points (time, position or chemical composition etc.). In MCR-ALS, a low-rank approximation of matrix $\mathbf{A}$ is obtained by solving the following equation:

$$
\mathbf{A}=\mathbf{W H},
$$

where $\mathbf{W}$ is a $m \times k$ matrix whose columns represent pure component spectra and $\mathbf{H}$ is a $k \times n$ matrix whose rows represent intensity profiles of corresponding pure spectral components (Fig. 1). The parameter $k$ defines the number of components in the constructed model, and is determined a priori by the user. An appropriate number of components can be best estimated beforehand either from singular value decomposition (SVD) or principal component analysis (PCA). However the number obtained from SVD or PCA should not be considered a fixed value, and models with slightly different numbers (both smaller and larger) should be constructed and evaluated before making a final decision on the accuracy of the analytical model.

In general, $\mathbf{W}$ and $\mathbf{H}$ are iteratively refined using alternating least squares so that the Frobenius norm $\|\mathbf{A}-\mathbf{W H}\|^{2}$ is minimized. Additionally, non-negative constraints on both $\mathbf{W}$ and $\mathbf{H}$ matrices $(\mathbf{W} \geq 0 ; \mathbf{H} \geq 0)$ are also imposed, since neither the Raman spectra nor its concentration profiles can take negative values in practical terms. Therefore, MCR-ALS analysis, which only requires non-negative constraints, has huge advantages over other factorization methods, such as SVD or PCA (require orthogonality of each component) in spectral analysis and provides physically meaningful results that can be interpreted in a straightforward manner.

Given the complexity in decomposing spectral components from biological samples (e.g., cells and tissues) and low signalto-noise Raman data (especially in imaging data sets), it is desired to improve the prediction accuracy and the interpretability of the model. In this regard, Lasso regression (L1-norm; $\alpha$ ) can be used:

$$
\begin{aligned}
& \left(\mathbf{W}^{\mathrm{T}} \mathbf{W}+\alpha^{2} \mathbf{E}\right) \mathbf{H}=\mathbf{W}^{\mathrm{T}} \mathbf{A}, \\
& \left(\mathbf{H H}^{\mathrm{T}}+\alpha^{2} \mathbf{E}\right) \mathbf{W}=\mathbf{H} \mathbf{A}^{\mathrm{T}},
\end{aligned}
$$

where $\mathbf{E}$ is a $k \times k$ matrix whose elements are all unity. An additional penalty such as ridge regression (L2-norm; $\beta$ ), is also commonly used to obtain a sparser solution: 

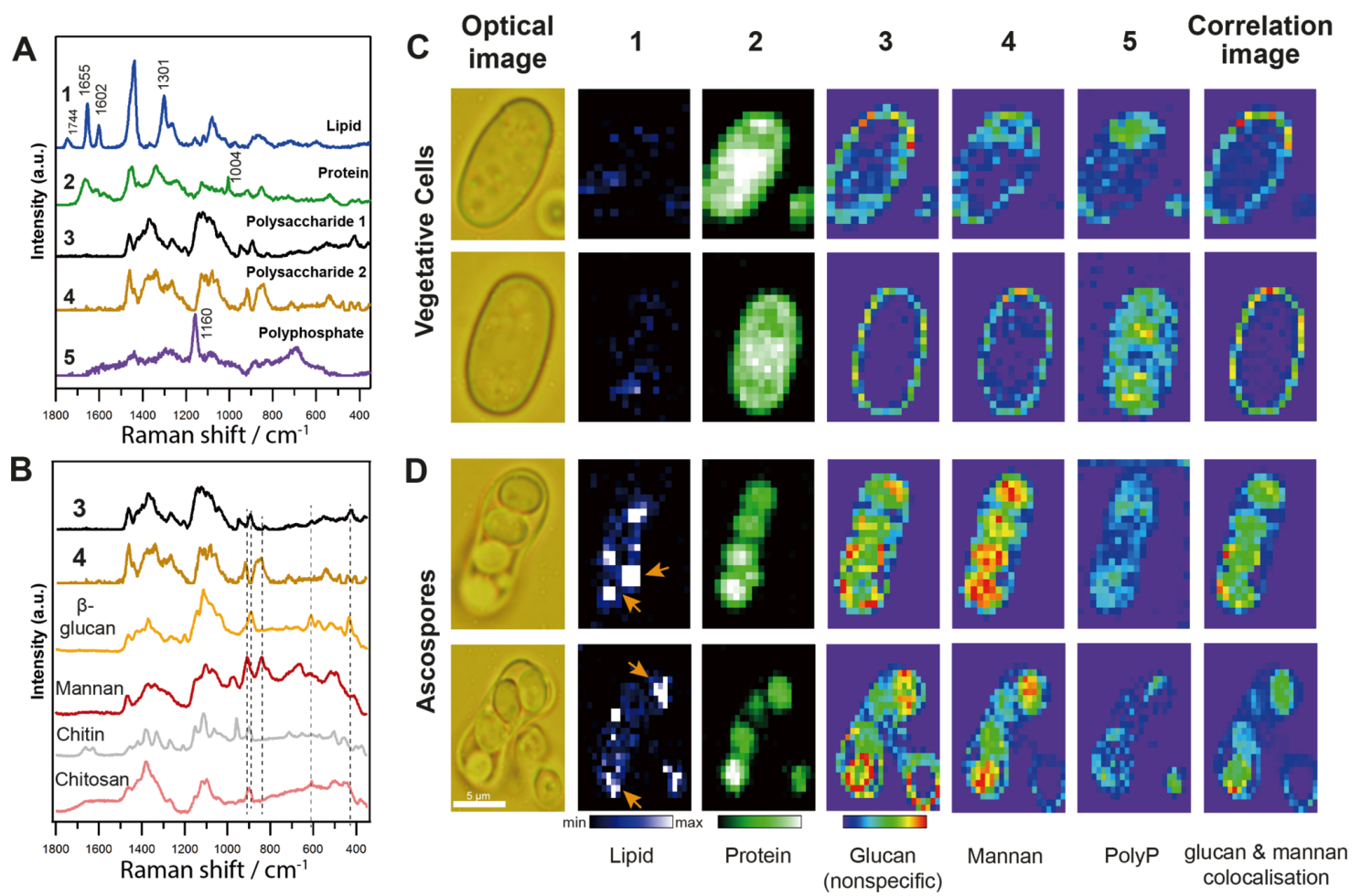

Fig. 2 MCR-ALS results obtained from Raman imaging of fission yeast vegetative cells and ascospores. (A) Pure component Raman spectra, (B) comparison of MCR components 3 and 4 with standard polysaccharide Raman spectra, (C) corresponding MCR component distribution images from vegetative cells and (D) ascospores. Adapted from Ref. 39.

$$
\begin{aligned}
& \left(\mathbf{W}^{\mathrm{T}} \mathbf{W}+\beta^{2} \mathbf{I}\right) \mathbf{H}=\mathbf{W}^{\mathrm{T}} \mathbf{A}, \\
& \left(\mathbf{H H}^{\mathrm{T}}+\beta^{2} \mathbf{I}\right) \mathbf{W}=\mathbf{H A}^{\mathrm{T}},
\end{aligned}
$$

where $\mathbf{I}$ is a $k \times k$ identity matrix.

Initialization is also a key point to be considered while implementing the algorithm. Initial guesses for $\mathbf{W}$ and $\mathbf{H}$ can either be random non-negative values or be based on other methods, like SVD. Additionally, any known information about the spectral or concentration profiles can also be included before the analysis. Therefore, a few trials should be done with different initialization methods and number of components while keeping the experimental data in mind to gain experience. Eventually, appropriate parameters should be decided by the user in advance to construct a meaningful model.

\section{Cellular Applications}

\subsection{Cell wall polysaccharide imaging}

The cell wall, though absent in animal cells, plays key roles, such as providing rigidity, structural support and offers protection against mechanical stress in fungi, plants and prokaryotic cells. Most of the cell walls are composed of complex polysaccharide-based materials. Moreover, its composition, properties and form changes depending on the cell type, cell cycle stage and growth conditions. Adding to the complexity is the structural similarities of these polymers. Traditionally, many analytical and microscopic (optical and electron microscopy) methods have been utilized for understanding the cell wall structure and composition. However, these are either destructive and too specific, or lack chemical information, while also being expensive and time consuming.
Hence, it is important to develop methods to study multiple components of the cell wall simultaneously with high specificity. Raman micro-spectroscopy has been suggested as an alternative, since it provides complete chemical information with both specificity and high spatial resolution in a non-destructive manner. However, another level of complexity arises owing to high structural similarities of such matrix polymers, which lead to many overlapping Raman bands. Several multivariate techniques have been employed in this regard, but the success of MCR-ALS is overwhelming since it can provide interpretable pure spectral component along with their distribution maps from imaging data sets.

For example, fungal cell walls are medically important since they represent a drug target site for antifungal drugs. However, the fungal spore wall architecture is poorly understood due to the lack of methods that can identify and image multiple components via $\alpha$-glucan, $\beta$-glucan, mannan, chitin, chitosan etc. at the single cell level. Recently, we imaged fungal cell and spore wall components successfully by employing Raman microscopy in a fission yeast model, as shown in Fig. $2 .{ }^{42}$ With the help of MCR-ALS analysis, the pure component spectra of intracellular bio-macromolecules, such as proteins, lipids and a couple of polysaccharide components, were obtained (Fig. 2A). Comparisons of the polysaccharide components from MCR with pure chemical standards revealed them to be glucan and mannan polymers (Fig. 2B). Corresponding MCR analyzed Raman images showed quite different distribution maps in vegetative cells and spores. It was revealed that while the yeast vegetative cell wall was made up of both $\alpha$ - and $\beta$-glucans, the spore wall was exclusively made of $\alpha$-glucan. Additionally, from correlation images, mannan was found to be distributed primarily in spores, but was absent in the ascal wall (Figs. 2C and 2D). Such detailed information concerning the dynamic 

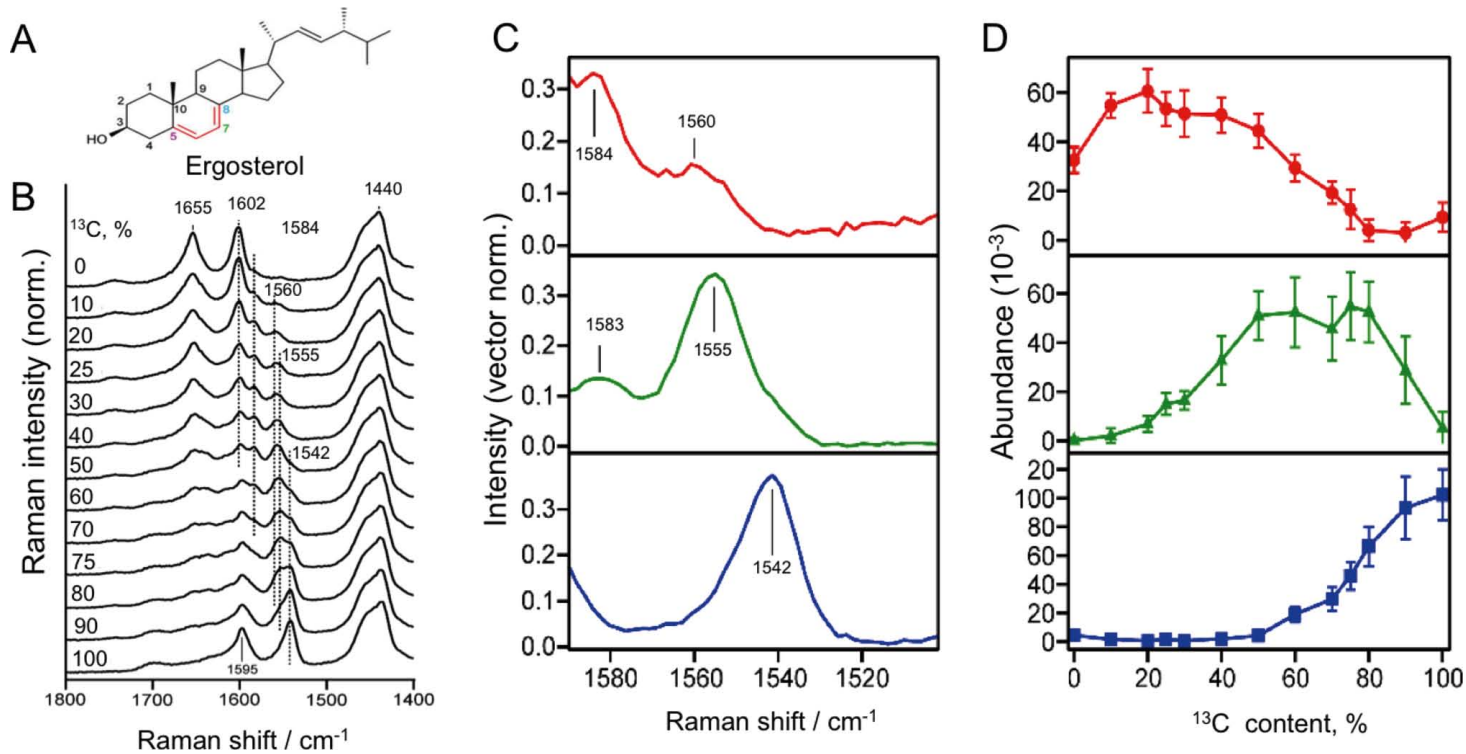

Fig. 3 MCR-ALS results from Raman spectroscopic study of ergosterol metabolism in fission yeast. (A) Molecular structure of ergosterol, (B) lipid Raman spectra with increasing ${ }^{13} \mathrm{C}$ concentration in medium, (C) three component MCR model resolved Raman spectra and (D) corresponding relative abundance profiles [red, mono ${ }^{13} \mathrm{C}$-substituted; green, di and tri ${ }^{13} \mathrm{C}$-substituted; blue, fully ${ }^{13} \mathrm{C}$ substituted]. Adapted with permission from Ref. 6. Copyrights (2014) American Chemical Society.

spore wall architecture can only be understood by applying MCR-ALS.

Similarly, plant cell wall architecture is also quite complex with primary and secondary layers. Major polysaccharides that make up these structures include cellulose, hemicellulose, pectin, lignin etc. Investigating the cell wall composition together with their structure at different stages is expected to reveal a comprehensive picture of biomolecular changes during plant cell wall development. In fact Raman spectroscopy has been utilized to study plant cell walls with high chemical specificity. ${ }^{43-46}$ However, MCR-ALS coupled approach is scarce. Chylinska et al. have demonstrated that it is possible to distinguish cellulose and pectins in the cell wall of tomato tissue from minimally prepared samples by employing MCR-ALS analysis. ${ }^{47}$ Moreover, their distributions were also visualized with high specificity, which is otherwise not possible using single band imaging. Recently, Liu et al. utilized hyperspectral stimulated Raman scattering microscopy coupled with MCRALS to reveal spatial distribution of functional groups of lignin in plant cell walls. ${ }^{48}$ Concentration maps of the aromatic ring, aldehyde $\mathrm{C}=\mathrm{C}$ and alcohol $\mathrm{C}=\mathrm{C}$ bonds were constructed from studies on wild-type and transgenic Arabidopsis plants. A further spatially distinct distribution of aldehyde and alcohol groups was found in the thickened secondary cell wall of bristle grass, a widely used biomass model. Real time monitoring of the reduction of aldehyde to alcohol has also been achieved in an intact plant tissue. These studies undoubtedly prove that Raman microscopy in combination with MCR-ALS can be very useful in understanding the dynamics of cell wall architecture.

\subsection{Single cell biochemistry}

Knowledge about the components of cells and how they work is central to our understanding of life. Much of what we understand in biology can be attributed to cell biology. Cells, being the smallest functional unit in an organism, can be used to study structure, organization, function of organelles driving the life cycle, which eventually helps to understand the whole organism at any given instant. Moreover studies on cellular metabolic processes are essential to biomedical research, especially for cancer and other diseases. Yet, they remain a major challenge in the current systems biology, and has led to extensive metabolomics research employing destructive methods. Hence, studying metabolic processes at the single cell level in a non-destructive manner is of paramount importance to accurately map the global structure of the complex cellular metabolic network at any given point in time. Recent advancements in nuclear magnetic resonance and mass spectrometry have led to powerful, stable isotope probing (SIP) based methods such as metabolic flux analysis, mass isotopomer distribution analysis and DNA-/RNA-based SIP in metabolomics research. Nevertheless all these methods either require destructive procedures or lack space specificity. Therefore, to probe metabolic processes, isotope labeled Raman microscopy has been suggested and developed as an alternative strategy to conventional biochemical and mass spectrometry based approaches as it is a non-destructive method with subcellular resolution. ${ }^{49,50}$

However, studying the series of steps involved in biochemical pathways using stable isotopes requires identification of isotopomers, and thus may not be a straightforward approach. It is this difficulty that led to exploring the possibility of coupling MCR-ALS to study metabolic pathways at the single cell level. Indeed, we have demonstrated that it is possible to study ergosterol metabolism in detail at the single cell level using fission yeast model. ${ }^{8}$ Figure 3 shows the space-resolved lipid Raman spectra of $S$. pombe in mixed ${ }^{12} \mathrm{C}$ and ${ }^{13} \mathrm{C}$ isotope media. The band at $1602 \mathrm{~cm}^{-1}$, corresponding to $\mathrm{C}=\mathrm{C}-\mathrm{C}=\mathrm{C}$ symmetric stretch of the 5,7-diene structure of ergosterol in a pure ${ }^{12} \mathrm{C}$ medium redshifts to $1542 \mathrm{~cm}^{-1}$ in the ${ }^{13} \mathrm{C}$ medium. In the case of isotope mixture media, intermediate bands representing various isotopomers can be observed at 1584, 1560 and $1555 \mathrm{~cm}^{-1}$ with increasing ${ }^{13} \mathrm{C}$ concentration (Fig. 3A). MCRALS analysis resolved 3 components (Fig. 3B), mono- ${ }^{13} \mathrm{C}-$ substituted, di- and tri- ${ }^{13} \mathrm{C}$-substituted and fully ${ }^{13} \mathrm{C}$-substituted respectively along with their relative abundances in relation to isotope mixture in the medium (Fig. 3C). Such possibilities are 

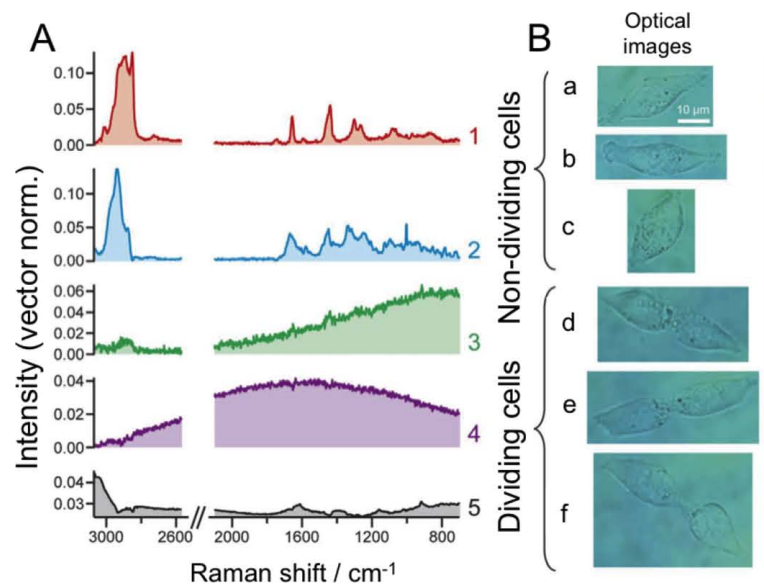

1

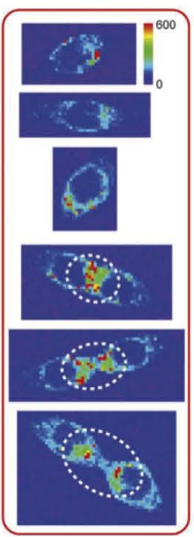

2

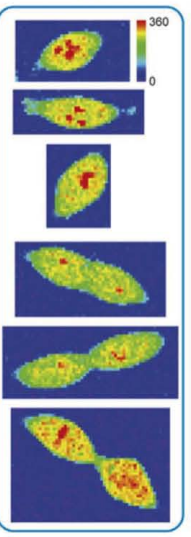

3

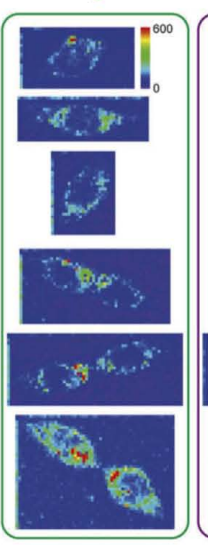

4

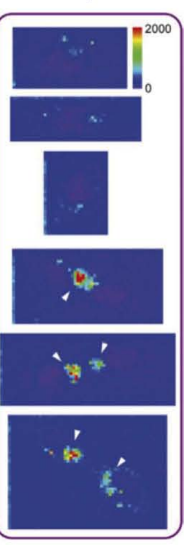

Fig. 4 MCR analysis results from HCT116 cells in interphase and cytokinesis. (A) MCR resolved intrinsic Raman spectra [1, lipid; 2, protein; 3 and 4, auto fluorescence; 5, medium]; (B) corresponding distribution images of non-dividing cells $[\mathrm{a}-\mathrm{c}]$ and dividing cells [d - f]. Adapted from Ref. 49.

expected to have important implications for the online monitoring of metabolites in industrial processes.

\section{3·3 Exploratory studies}

Another aspect of single cell biology focuses on understanding the molecular mechanism of the cell cycle, the complex and dynamic process that is central to reproduction in all living organisms. The sheer number of cellular components that work together in an orderly fashion to bring about cell division makes it difficult to obtain a holistic picture of this complex process. Again, Raman microspectroscopy coupled with MCR-ALS has proved to be a valuable tool to understand spatiotemporal changes at the single cell level. Huang et al. demonstrated MCR analysis on a four-dimensional (spectral dimension, two dimensions in space and time dimension) Raman data obtained from time-lapse Raman imaging of a single dividing yeast cell. ${ }^{51}$ This particular approach disentangled dynamic changes in both concentrations and distributions of several major intracellular bio-macromolecules, such as lipids, proteins and polysaccharides, during the cell division cycle in a straightforward manner. A significant decrease of lipids $(-50 \%)$ was observed after cell division. Additionally, a protein associated component indicating the phosphorylation event was also found indicating the superiority of this method over univariate approaches. Such exploratory studies are believed to help us to unravel yet unknown roles of key metabolites and underlying mechanisms.

Recently, Hsu et al. employed MCR for an exploratory investigation of cytokinesis at the molecular level in living cells whose results are showed in Fig. $4{ }^{52}$ The study on human colon cancer cells (HCT116) was aimed at unraveling novel chemical components involved in cytokinesis. Pure component Raman spectra of proteins and lipids along with their distribution maps in both interphase and dividing cells were obtained. Interestingly, MCR resolved an additional strong auto fluorescence component (component 4 in Fig. 4) which was found to accumulate predominantly near the cleavage furrow and colocalized with lipid component during cell division. This may serve as an endogenous probe for the in vivo visualization of the progress during cell division.

\section{Medical Applications}

Historically, Hartman et al. first reported Raman spectra of intact viruses in the early 70s, and revealed structural information related to viral protein and nucleic acid. ${ }^{53}$ In 1974, Yu et al. reported Raman spectra from an intact calf eye lens. ${ }^{54}$ Around the same time, Larsson et al. compared Raman spectra of blood from both healthy and diseased patients, and even proposed blood testing by Raman spectroscopy. ${ }^{55}$ These works immediately opened up lens research. Detailed studies by Ozaki group led to a deeper understanding of lens aging and cataract formation through lens hydration, intermolecular disulfide bridge formation and micro environment change of tyrosine residues in lens protein. ${ }^{56-61}$ Raman spectroscopy has been successfully employed to both in vivo and in vitro, and one can easily find its application in various fields of medicine including pathology, ${ }^{62,63}$ physiology ${ }^{64}$ virology ${ }^{65}$ urology, ${ }^{66,67}$ and dentistry. ${ }^{68-70}$ Either by using cells, dissected tissues or real time monitoring during surgery, researchers have demonstrated the utility of Raman spectroscopy, particularly in cancers related to brain,,$^{71,72}$ breast, ${ }^{73,74}$ lungs, ${ }^{75,76}$ skin, ${ }^{77,78}$ oesophagus, ${ }^{79,80}$ prostates $^{81,82}$ and bone diseases. ${ }^{83,84}$ Additionally, by analyzing biofluids such as blood and urine, non-invasive diagnostic assays are also being actively developed for many diseases such as diabetes (glucose level monitoring), ${ }^{85,86}$ cancer, ${ }^{87,88}$ asthma, ${ }^{89}$ and malaria. ${ }^{90,91}$ Most of these studies relied on univariate analysis (one or two marker bands used for biomolecular identification). However, in order to obtain both qualitative and quantitative information, it is important to separate pure component spectra.

In a recent work, Patel et al. employed MCR-ALS to Raman imaging data from benign and tumorigenic uterine tissues..$^{25}$ The results demonstrated the superiority of MCR-ALS over other methods (HCA and PCA) in obtaining high contrast images with clear and defined margins of histological features. This is especially useful during surgical excision where there is a need to remove positive tumor margins. Figure 5 shows MCR component images of benign (tumor-adjacent) endometrium, stage I and stage II endometrioid cancer. Application of MCR to such a complex specimen led to accurate demarcation of features associated with glandular epithelium, stroma, glandular lumen and myometrium. Such a method is believed to remove doubts about cancer margin, and to provide a high degree of confidence during surgery, which can in turn help to decrease the risk of the local recurrence of cancer.

Another interesting demonstration was achieved recently by Chen et al., in which they have developed an automatic and 


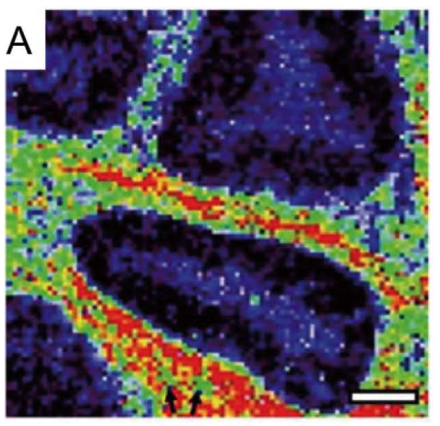

B

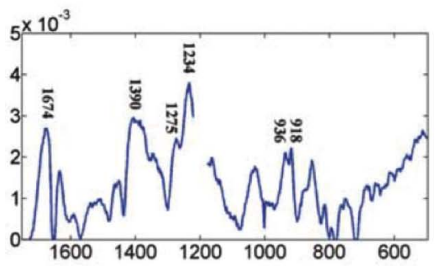

Raman shift / $\mathrm{cm}^{-1}$

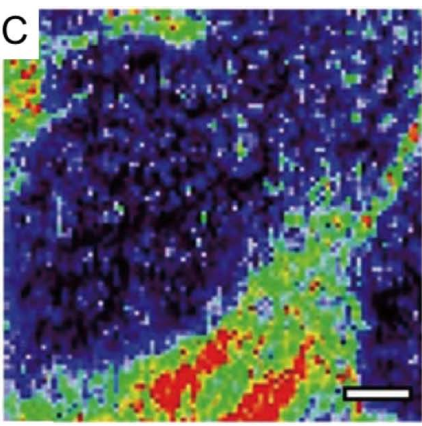

$\mathrm{D}$

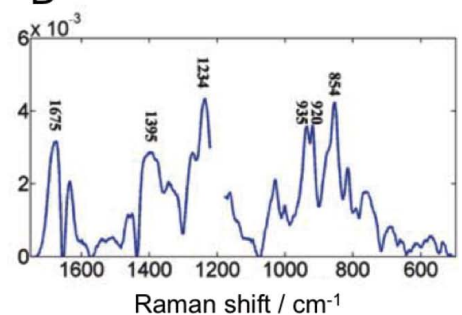

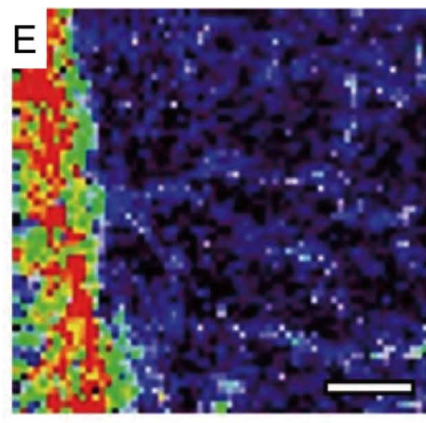

F

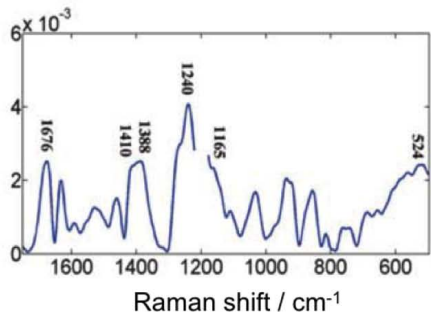

Fig. 5 MCR results from Raman imaging on benign and tumourigenic uterine tissues. MCR resolved distribution image and corresponding spectral profile of $(\mathrm{A}-\mathrm{B})$ benign endometrial tissue, $(\mathrm{C}-\mathrm{D})$ stage IA endometrioid cancer and $(\mathrm{E}-\mathrm{F})$ stage IIA endometrioid cancer. Scale bar $=50 \mu \mathrm{m}$. Color scale: black $<<$ green $<<$ red. Adapted from Ref. 22 with permission from The Royal Society of Chemistry.

A $\frac{a}{1}$

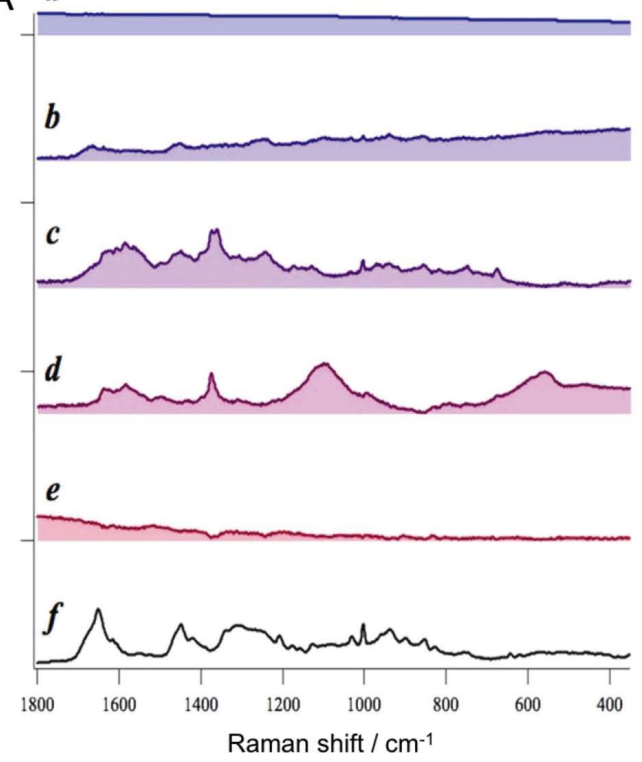

B

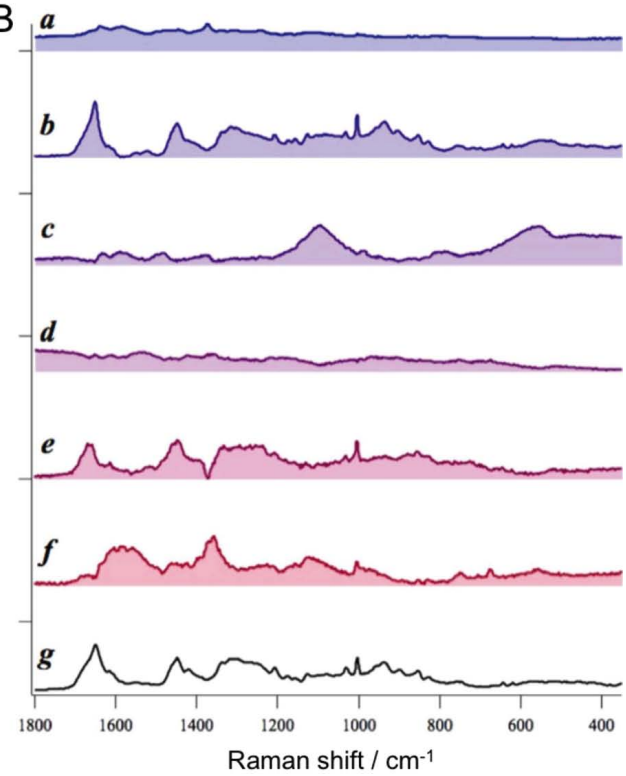

Fig. 6 MCR analysis results from normal and cancerous tissues. Intrinsic spectral components (A) normal tissue sample [a - e] compared with standard keratin spectrum [f] from a patient and (B) oral squamous cell carcinoma tissue from the same patient $[\mathrm{a}-\mathrm{f}]$ compared with standard keratin spectrum [g]. Adapted from Ref. 17.

objective oral cancer diagnosis by Raman spectroscopy and MCR. Raman spectra from both normal and cancerous tissue of a patient were obtained and MCR was constructed (Fig. 6). ${ }^{20}$ By making use of keratin, a well-established molecular marker of oral squamous cell carcinoma (OSCC), discrimination between normal and oral cancerous tissue was automated, primarily by comparing the MCR analyzed components with the standard keratin Raman spectrum. The Raman spectroscopic method for molecular monitoring of epithelial to mesenchymal transition, an indication of metastasis progression, in breast cancer cells has also been established. ${ }^{92}$ MCR performed on confluent and sparse breast cancer cells showed a decrease in the cytokeratin content, and an increase in the fatty acid and tryptophan content indicating the transition.

The dynamic monitoring of retina inflammation has been demonstrated as well by in vivo Raman spectroscopy coupled with MCR. ${ }^{93}$ Dynamic changes of molecular components such as increase of immune mediators, changes in energy producing molecules and decrease of phosphatidylcholine were observed during the evolution of retina inflammation which may become 
a promising application to study retina disease in vivo.

To develop successful biofluids-based Raman assays, the concentration of metabolite plays a key role. Surface-enhanced Raman spectroscopy (SERS) can be a useful alternative to detect for low concentration metabolites, and when combined with MCR it becomes even more powerful, since it renders pure component separation. Poppi lab has utilized this strategy to identify and possibly quantify different metabolites. ${ }^{94-97}$ A quantitative method for an on-site determination of uric acid in urine using gold nanoparticle coated paper substrate for SERS detection has been suggested. ${ }^{96}$ Also, by using a similar nanoparticle coated substrate, a procedure for the determination of the antibiotic moxifloxacin in urine has been demonstrated. In these methods, standard addition in conjunction to MCR were applied to eliminate the matrix effects and to address overlapping bands, thereby isolating the spectral contribution of the analyte in the presence unexpected interferences from urine.

\section{Conclusions and Outlooks}

Raman spectroscopy by itself has been well established as a valuable technique, especially in biological and medical contexts. However, it becomes even more powerful when coupled with MCR-ALS analysis, as can be realized from examples in this review. Though utilization of MCR in Raman spectroscopy is considered to be in its early stage, the volume of literature elucidating structural and functional relationships of biomolecular components is steadily growing. Needless to mention, there is a huge potential for this method to advance our understanding in fundamental biology, and will also be of great use in in vivo and in vitro disease diagnostic applications. It is only appropriate to say that MCR-ALS coupled Raman spectroscopy is a sleeping giant waiting to unravel the mysteries in biological and medical sciences.

\section{Acknowledgements}

A part of this work was supported by Grants-in-Aid for scientific research by JSPS to T. Y. (2431002, 26650133 and 15H04763). The authors would like to thank Prof. Kawamukai of Shimane University for supplying required fission yeast samples and for his guidance in studying them.

\section{References}

1. Y. S. Huang, T. Karashima, M. Yamamoto, and $\mathrm{H}$. Hamaguchi, Biochemistry, 2005, 44, 10009.

2. Y. S. Huang, T. Karashima, M. Yamamoto, T. Ogura, and H. Hamaguchi, J. Raman Spectrosc., 2004, 35, 525.

3. H. N. Noothalapati Venkata and S. Shigeto, Chem. Biol., 2012, 19, 1373.

4. M. Kakita, V. Kaliaperumal, and H. Hamaguchi, J. Biophotonics, 2012, 5, 20.

5. M. Kakita, M. Okuno, and H. Hamaguchi, J. Biophotonics, 2013, 6, 256.

6. H. Segawa, M. Okuno, P. Leproux, V. Couderc, T. Ozawa, and H. Kano, Anal. Sci., 2015, 31, 299.

7. H. Takeuchi, Anal. Sci., 2011, 27, 1077.

8. H. Noothalapati and S. Shigeto, Anal. Chem., 2014, 86, 7828.

9. M. Li, W. E. Huang, C. M. Gibson, P. W. Fowler, and A. Jousset, Anal. Chem., 2013, 85, 1642.

10. K. Maquelin, C. Kirschner, L. P. Choo-Smith, N. van den
Braak, H. P. Endtz, D. Naumann, and G. J. Puppels, J. Microbiol. Methods, 2002, 51, 255.

11. U. Neugebauer, J. H. Clement, T. Bocklitz, C. Krafft, and J. Popp, J. Biophotonics, 2010, 3, 579.

12. P. C. Buijtels, H. F. Willemse-Erix, P. L. Petit, H. P. Endtz, G. J. Puppels, H. A. Verbrugh, A. van Belkum, D. van Soolingen, and K. Maquelin, J. Clin. Microbiol., 2008, 46, 961.

13. M. Knauer, N. P. Ivleva, R. Niessner, and C. Haisch, Anal. Sci., 2010, 26, 761.

14. A. Beljebbar, O. Bouche, M. D. Diebold, P. J. Guillou, J. P. Palot, D. Eudes, and M. Manfait, Crit. Rev. Oncol. Hematol., 2009, 72, 255.

15. P. Matousek, E. R. Draper, A. E. Goodship, I. P. Clark, K. L. Ronayne, and A. W. Parker, Appl. Spectrosc., 2006, 60, 758.

16. H. Noothalapati, S. Uemura, N. Ohshima, Y. Kinoshita, M. Ando, H. Hamaguchi, and T. Yamamoto, Vib. Spectrosc., 2016, $85,7$.

17. P. Matousek and N. Stone, Chem. Soc. Rev., 2016, 45, 1794.

18. M. Jermyn, K. Mok, J. Mercier, J. Desroches, J. Pichette, K. Saint-Arnaud, L. Bernstein, M. C. Guiot, K. Petrecca, and F. Leblond, Sci. Trans. Med., 2015, 7, 274 ra19.

19. A. Nijssen, K. Maquelin, L. F. Santos, P. J. Caspers, T. C. Bakker Schut, J. C. den Hollander, M. H. Neumann, and G. J. Puppels, J. Biomed. Opt., 2007, 12, 034004.

20. P. H. Chen, R. Shimada, S. Yabumoto, H. Okajima, M. Ando, C. T. Chang, L. T. Lee, Y. K. Wong, A. Chiou, and H. Hamaguchi, Sci. Rep., 2016, 6, 20097.

21. C. A. Drumm and M. D. Morris, Appl. Spectrosc., 1995, 49, 1331.

22. M. V. Chowdary, K. K. Kumar, J. Kurien, S. Mathew, and C. M. Krishna, Biopolymers, 2006, 83, 556.

23. J. L. Pichardo-Molina, C. Frausto-Reyes, O. BarbosaGarcia, R. Huerta-Franco, J. L. Gonzalez-Trujillo, C. A. Ramirez-Alvarado, G. Gutierrez-Juarez, and C. MedinaGutierrez, Lasers Med. Sci., 2007, 22, 229.

24. B. Bodanese, F. L. Silveira, R. A. Zangaro, M. T. Pacheco, C. A. Pasqualucci, and L. Silveira, Jr., Photomed. Laser Surg., 2012, 30, 381.

25. I. I. Patel, J. Trevisan, G. Evans, V. Llabjani, P. L. MartinHirsch, H. F. Stringfellow, and F. L. Martin, Analyst, 2011, 136, 4950.

26. M. Ando and H. Hamaguchi, J. Biomed. Opt., 2014, 19, 011016.

27. M. Vosough and H. M. Esfahani, Talanta, 2013, 113, 68.

28. A. Mancha de Llanos, M. M. De Zan, M. J. Culzoni, A. Espinosa-Mansilla, F. Canada-Canada, A. Munoz de la Pena, and H. C. Goicoechea, Anal. Bioanal. Chem., 2011, 399, 2123.

29. M. Vosough and N. R. Mojdehi, Talanta, 2011, 85, 2175.

30. P. Jonsson, E. S. Johansson, A. Wuolikainen, J. Lindberg, I. Schuppe-Koistinen, M. Kusano, M. Sjostrom, J. Trygg, T. Moritz, and H. Antti, J. Proteome Res., 2006, 5, 1407.

31. M. Vosough and A. Salemi, Talanta, 2007, 73, 30.

32. M. Jalali-Heravi and H. Parastar, Talanta, 2011, 85, 835.

33. N. E. Llamas, M. Garrido, M. S. Di Nezio, and B. S. Fernandez Band, Anal. Chim. Acta, 2009, 655, 38.

34. J. Saurina, S. Hernandez-Cassou, and R. Tauler, Anal. Chem., 1997, 69, 2329.

35. A. Kandelbauer, W. Kessler, and R. W. Kessler, Anal. Bioanal. Chem., 2008, 390, 1303.

36. L. Blanchet, A. Mezzetti, C. Ruckebusch, J. P. Huvenne, and A. de Juan, Anal. Bioanal. Chem., 2007, 387, 1863.

37. O. Abbas, C. Rebufa, N. Dupuy, and J. Kister, Talanta, 2008, 77, 200. 
38. V. del Rio, M. P. Callao, M. S. Larrechi, L. Montero de Espinosa, J. C. Ronda, and V. Cadiz, Anal. Chim. Acta, 2009, 642, 148.

39. J. C. Esteves da Silva and R. Tauler, Appl. Spectrosc., 2006, 60, 1315.

40. P. Kumar, A. Verma, S. Maiti, R. Gargallo, and S. Chowdhury, Biochemistry, 2005, 44, 16426.

41. S. Navea, A. de Juan, and R. Tauler, Anal. Chem., 2002, 74, 6031.

42. H. Noothalapati, T. Sasaki, T. Kaino, M. Kawamukai, M. Ando, H. Hamaguchi, and T. Yamamoto, Sci. Rep., 2016, 6, 27789.

43. R. H. Atalla and U. P. Agarwal, Science, 1985, 227, 636.

44. N. Gierlinger and M. Schwanninger, Plant Physiol., 2006, 140, 1246.

45. N. Gierlinger, T. Keplinger, and M. Harrington, Nat. Protocol., 2012, 7, 1694.

46. T. Hanninen, E. Kontturi, and T. Vuorinen, Phytochemistry, 2011, 72, 1889.

47. M. Chylinska, M. Szymanska-Chargot, and A. Zdunek, Plant Methods, 2014, 10, 14.

48. B. Liu, P. Wang, J. I. Kim, D. Zhang, Y. Xia, C. Chapple, and J. X. Cheng, Anal. Chem., 2015, 87, 9436.

49. H. Noothalapati and S. Shigeto, Chem. Biol., 2012, 19, 1373.

50. B. N. V. Kumar, S. Guo, T. Bocklitz, P. Rosch, and J. Popp, Anal. Chem., 2016, 88, 7574.

51. C. K. Huang, M. Ando, H. O. Hamaguchi, and S. Shigeto, Anal. Chem., 2012, 84, 5661.

52. J. F. Hsu, P. Y. Hsieh, H. Y. Hsu, and S. Shigeto, Sci. Rep., 2015, 5, 17541.

53. K. A. Hartman, N. Clayton, and G. J. Thomas, Jr., Biochem. Biophys. Res. Commun., 1973, 50, 942.

54. N. T. Yu, B. H. Jo, R. C. Chang, and J. D. Huber, Arch. Biochem. Biophys., 1974, 160, 614.

55. K. Larsson and L. Hellgren, Experientia, 1974, 30, 481.

56. A. Mizuno, Y. Ozaki, Y. Kamada, H. Miyazaki, K. Itoh, and K. Iriyama, Curr. Eye Res., 1981, 1, 609.

57. K. Iriyama, A. Mizuno, Y. Ozaki, K. Itoh, and H. Matsuzaki, Curr. Eye Res., 1982, 2, 489.

58. Y. Ozaki, A. Mizuno, K. Itoh, M. Yoshiura, T. Iwamoto, and K. Iriyama, Biochemistry, 1983, 22, 6254.

59. K. Itoh, Y. Ozaki, A. Mizuno, and K. Iriyama, Biochemistry, 1983, 22, 1773.

60. A. Mizuno, Y. Ozaki, K. Itoh, S. Matsushima, and K. Iriyama, Biochem. Biophys. Res. Commun., 1984, 119, 989.

61. Y. Ozaki, A. Mizuno, K. Itoh, and K. Iriyama, J. Biol. Chem., 1987, 262, 15545.

62. C. M. Krishna, G. D. Sockalingum, J. Kurien, L. Rao, L. Venteo, M. Pluot, M. Manfait, and V. B. Kartha, Appl. Spectrosc., 2004, 58, 1128.

63. K. Das, N. Stone, C. Kendall, C. Fowler, and J. ChristieBrown, Lasers Med. Sci., 2006, 21, 192.

64. L. Franzen and M. Windbergs, Adv. Drug Delivery Rev., 2015, 89, 91.

65. P. J. Lambert, A. G. Whitman, O. F. Dyson, and S. M. Akula, Virol. J., 2006, 3, 51.

66. V. S. Hanchanale, A. R. Rao, and S. Das, Indian J. Urol., 2008, 24, 444.

67. E. C. Osterberg, M. A. Laudano, and P. S. Li, Transl. Androl. Urol., 2014, 3, 84.

68. J. L. Gonzalez-Solis, E. Martinez-Cano, and Y. MaganaLopez, Laser Med. Sci., 2015, 30, 1675.

69. A. C. Ko, L. P. Choo-Smith, M. Hewko, M. G. Sowa, C. C. Dong, and B. Cleghorn, Opt. Express, 2006, 14, 203.

70. H. Tsuda and J. Arends, Adv. Dent. Res., 1997, 11, 539.
71. M. Kirsch, G. Schackert, R. Salzer, and C. Krafft, Anal. Bioanal. Chem., 2010, 398, 1707.

72. N. Bergner, A. Medyukhina, K. D. Geiger, M. Kirsch, G. Schackert, C. Krafft, and J. Popp, Anal. Bioanal. Chem., 2013, 405, 8719.

73. A. S. Haka, K. E. Shafer-Peltier, M. Fitzmaurice, J. Crowe, R. R. Dasari, and M. S. Feld, Proc. Natl. Acad. Sci. U. S. A., 2005, 102, 12371.

74. A. S. Haka, Z. Volynskaya, J. A. Gardecki, J. Nazemi, J. Lyons, D. Hicks, M. Fitzmaurice, R. R. Dasari, J. P. Crowe, and M. S. Feld, Cancer Res., 2006, 66, 3317.

75. Z. Huang, A. McWilliams, H. Lui, D. I. McLean, S. Lam, and H. Zeng, Int. J. Cancer, 2003, 107, 1047.

76. Y. Oshima, H. Shinzawa, T. Takenaka, C. Furihata, and H. Sato, J. Biomed. Opt., 2010, 15, 017009.

77. J. Zhao, H. Lui, D. I. McLean, and H. Zeng, Conf. Proc. IEEE Eng. Med. Biol. Soc., 2008, 2008, 3107.

78. H. Lui, J. Zhao, D. McLean, and H. Zeng, Cancer Res., 2012, 72, 2491.

79. C. Kendall, N. Stone, N. Shepherd, K. Geboes, B. Warren, R. Bennett, and H. Barr, J. Pathol., 2003, 200, 602.

80. G. Shetty, C. Kendall, N. Shepherd, N. Stone, and H. Barr, Br. J. Cancer, 2006, 94, 1460.

81. P. Crow, A. Molckovsky, N. Stone, J. Uff, B. Wilson, and L. M. WongKeeSong, Urology, 2005, 65, 1126.

82. M. C. Prieto, P. Matousek, M. Towrie, A. W. Parker, M. Wright, A. W. Ritchie, and N. Stone, J. Biomed. Opt., 2005, 10, 44006.

83. P. A. Felice, B. Gong, S. Ahsan, S. S. Deshpande, N. S. Nelson, A. Donneys, C. Tchanque-Fossuo, M. D. Morris, and S. R. Buchman, J. Bone Miner. Metab., 2015, 33, 279.

84. P. I. Okagbare, D. Begun, M. Tecklenburg, A. Awonusi, S. A. Goldstein, and M. D. Morris, J. Biomed. Opt., 2012, 17, 90502.

85. A. M. Enejder, T. G. Scecina, J. Oh, M. Hunter, W. C. Shih, S. Sasic, G. L. Horowitz, and M. S. Feld, J. Biomed. Opt., 2005, 10, 031114.

86. O. Lyandres, J. M. Yuen, N. C. Shah, R. P. VanDuyne, J. T. Walsh, and M. R. Glucksberg, Diabetes Technol. Ther, 2008, 10, 257.

87. E. Canetta, M. Mazilu, A. C. De Luca, A. E. Carruthers, K. Dholakia, S. Neilson, H. Sargeant, T. Briscoe, C. S. Herrington, and A. C. Riches, J. Biomed. Opt., 2011, 16, 037002 .

88. T. Bhattacharjee, A. Khan, G. Maru, A. Ingle, and C. M. Krishna, Analyst, 2015, 140, 456.

89. A. Sahu, K. Dalal, S. Naglot, P. Aggarwal, and C. Murali Krishna, PLoS One, 2013, 8, e78921.

90. K. Chen, C. Yuen, Y. Aniweh, P. Preiser, and Q. Liu, Sci. Rep., 2016, 6, 20177.

91. B. R. Wood and D. McNaughton, Expert Rev. Proteomics, 2006, 3, 525.

92. M. Marro, C. Nieva, R. Sanz-Pamplona, and A. Sierra, Biochim. Biophys. Acta, 2014, 1843, 1785.

93. M. Marro, A. Taubes, A. Abernathy, S. Balint, B. Moreno, B. Sanchez-Dalmau, E. H. Martinez-Lapiscina, I. AmatRoldan, D. Petrov, and P. Villoslada, J. Biophotonics, 2014, 7, 724.

94. C. D. Albuquerque and R. J. Poppi, Anal. Chim. Acta, 2015, 879, 24.

95. M. B. Mamian-Lopez and R. J. Poppi, Anal. Chim. Acta, 2013, 760, 53.

96. J. E. Villa and R. J. Poppi, Analyst, 2016, 2, 1966.

97. M. B. Mamian-Lopez and R. J. Poppi, Anal. Bioanal. Chem., 2013, 405, 7671. 